\title{
Research on Identification of Dust Particles on COF
}

\author{
Jiayi Zhang ${ }^{1}$, Qiang $\mathrm{Gao}^{2, a}$ \\ ${ }^{1}$ School of Mechanical and Electric Engineering, Soochow University, 215021 Su Zhou, China \\ ${ }^{2}$ School of Mechanical and Electric Engineering, Soochow University, 215021 Su Zhou, China
}

\begin{abstract}
Chip On Film(COF) is the key component of electronic products, and is different from Printed Circuit Board(PCB). The properties of high flexibility, thin thickness, lightweight and high wiring density make it difficult to inspect COF, especially dust particles interference. Dust particles are similar to defects, and it is hard to identify dust particles from defects, so dust particles interference of quality test is the difficulty of automatic surface defect detection. In this paper, a new method to identify dust particles is discussed from abnormal area called junction points detection and machine learning method Support Vector Machine(SVM) according to the characteristics of dust particles. As a result, a $94.8 \%$ correct rate of dust particles images identification has been achieved with the method.
\end{abstract}

Key Words. COF, Dust, Identification, Junction Point, SVM

\section{Introduction}

COF is a product using IC chip placing and flexible substrate carrying technologies ${ }^{[1]}$, as shown in Figure 1. COF with $25 \mu \mathrm{m}$ pitch was achieved by using a new etching technique ${ }^{[2]}$, therefore, COF meets light, thin, short and small design requirements of electrical products, and is a typical product of micro-nano manufacturing.

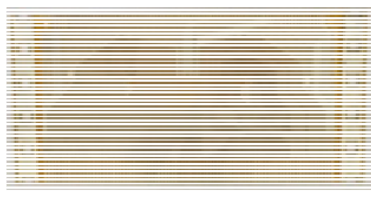

1) Front side

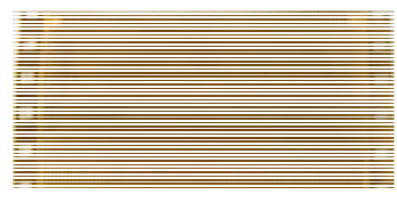

2) Back side
Figure 1. Chip On Film.

With the rapid development of Information Times, the properties of high flexibility, thin thickness, lightweight and high wiring density make the demand for COF enhance greatly, at the same time, the demand for automatic test of COF is higher and higher. Currently, quality test of COF is most completed by artificial, and it has high labor intensity and strong subjective interference ${ }^{[3]}$, especially for dust particles identification. While there are AOI(Automatic optical Inspection) equipments used in the quality detection of $\mathrm{COF}$, but dust particles are often as a defect detection error, which greatly reduces the production efficiency.

For dust particles identification, some researchers conducted some corresponding exploration. YoungJun Roh etc. put forward using the fast Fourier transform and fast Fourier inverse transformation technologies for reference images, operating subtraction on original image and reference image, then making segmentation on result

\footnotetext{
${ }^{a}$ Corresponding author: gaoqiang@suda.edu.cn
}

image from subtraction operation, extracting data from segmentation image with analysis of margin and intensity, and setting up Bayesian model for identification. However, the method is only applicable to linear circuit, cannot be applied to curved lines ${ }^{[4]}$. Rambabu K etc. proposed a method using template matching and analysis of many directions ${ }^{[5]}$, but contour of dust particles is complex, and dust particles have different gray levels, which makes determining a template difficult, analyzing many directions complex, and realizing the method hard. Chia-Te Liao etc. prenented a statistical learning method on the basis of SVM, but reference images are needed. In addition to this, the method involves image registration and Hausdorff distance calculation, requirement of accuracy is high, and amount of calculation is large ${ }^{[6]}$. Jin-soo Kim proposed a method based on reference images and parameters extraction from local binary pattern with location correction technology, which requires high accuracy ${ }^{[7]}$. Some of methods mentioned above are only suitable for linear circuit, and the other methods refer to reference image matching, position correction and similarity calculation technologies, which are realized intricately and whose calculation amount is large. According to these limitations and that papers about surface defect detection of COF have little been published, a dust particles identification method without reference images is put forward on the basis of junction point detection and SVM in this paper, which provides a new method for solving dust particles interference in the quality inspection process.

\section{Dust Particles Identification Algorithm}

Irregular contour, different gray levels and uncertain position of dust particles lead to that the traditional image segmentation technique is unable to achieve segmentation 
of dust particles. The image contrast technology requires reference images and high accuracy, so it is demanding. According to the characteristic that boundary of dust particles is irregular in this paper, a method without reference images combining abnormal region detection through geometrical junction point and identification by using SVM algorithm is proposed.

The information of gray images is complex, and gray levels of different images are different, especially $\mathrm{Cu}$ conductors because of light diffraction, mechanical vibration and illumination variation during the image acquisition process. Therefore, the adaptive threshold method is adopted to make original images segmentation to achieve binary images that are simple and do not change the state of dust particles' boundary, the original dust particles images are show in Figure 2, and the binary images are shown in Figure 3.

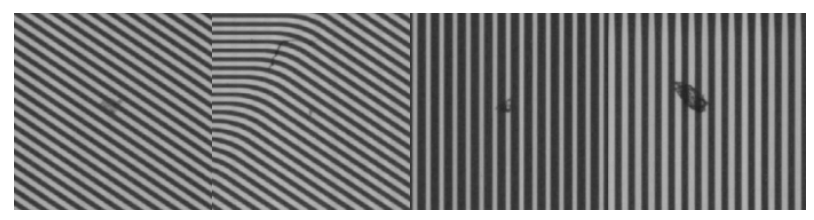

Figure 2. Original Dust Particles Images.

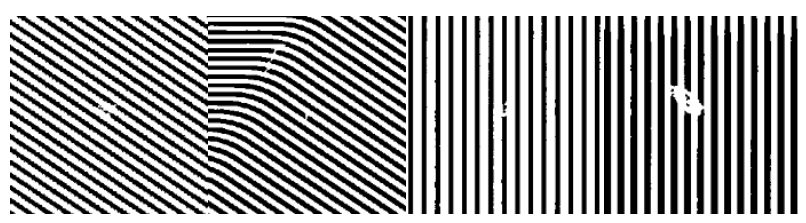

Figure 3. Binary Images.

Skeleton as a thinning structure, is the important topological description of target image, and keeps original shape of the binary image containing important shape information. The essence of thinning: objects in the image are simplified to single pixel width line, which shows its topological properties ${ }^{[8]}$. Skeleton extraction is based on image thinning algorithm ${ }^{[9]}$, and look-up table thinning algorithm and layer by layer thinning algorithm are used commonly ${ }^{[10]}$. In this paper, the look-up table thinning algorithm based on the eight neighborhood is applied to extract the skeleton ${ }^{[11]}$.

A junction point is defined as the point at the position, where two skeleton lines cross, and the angular difference is greater than zero ${ }^{[12]}$. The flow chart of the junction point detection is shown in Figure 4. The result of skeleton extraction is shown in Figure 5, and the white part is skeleton. The result of the junction point detection is shown in Figure 6, and the white points are junction points in skeleton images. The result of the junction point detection in dust region is shown in Figure 7, and the red points are junction points, which indicates that junction points exist in dust particles.

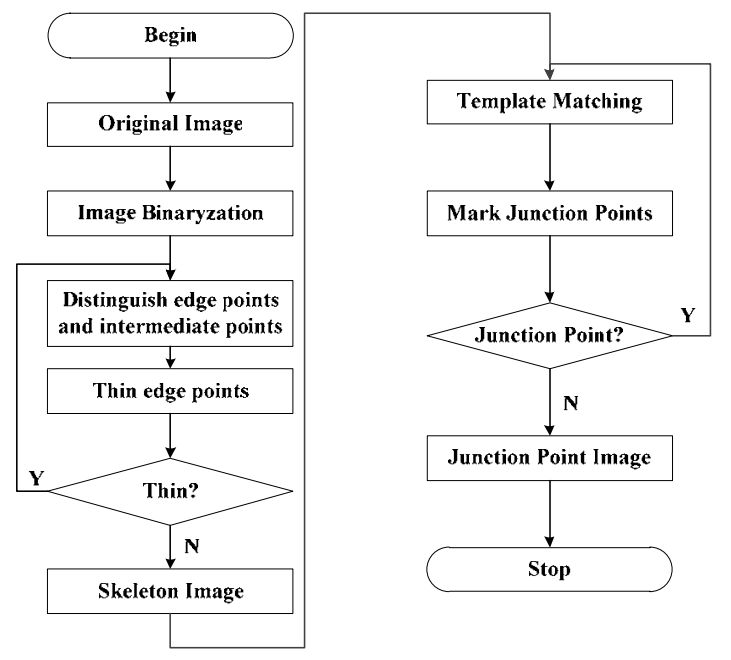

Figure 4. Junction Points Detection Flow Chart.

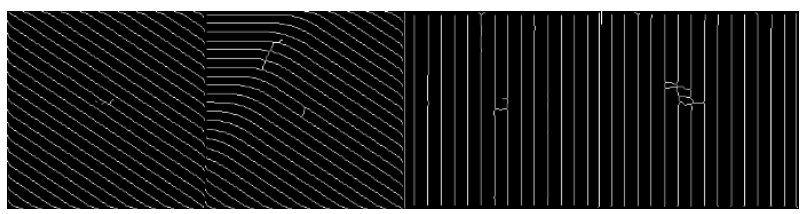

Figure 5. Skeleton Images.

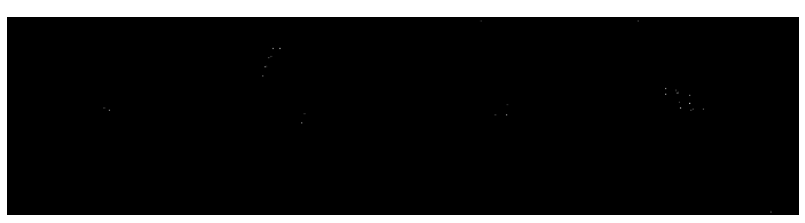

Figure 6. Junction Point Images.

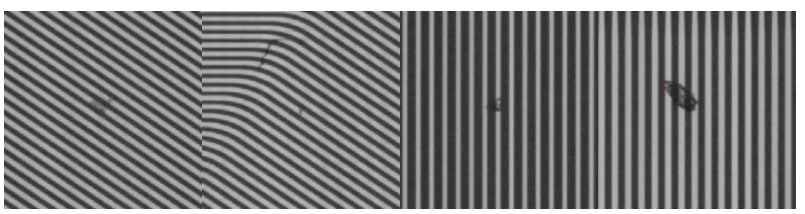

Figure 7. Junction Points in Dust Region.

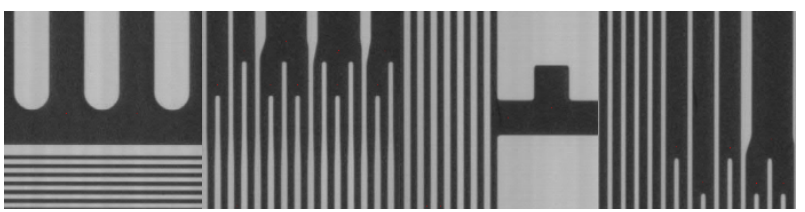

Figure 8. Normal Images with Junction Points.

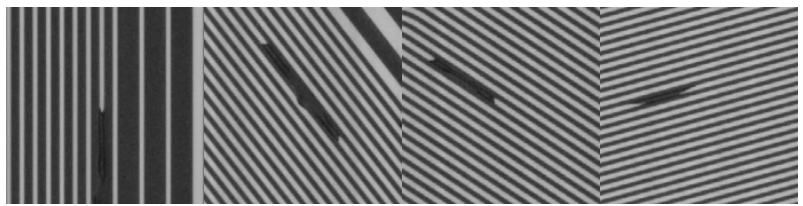

Figure 9. Defect Images with Junction Points.

Figure 7 shows that junction points exist in dust particles, but some normal images and defect images also have the same feature. Some normal images with junction points are shown in Figure 8, and some defect images with junction points are shown in Figure 9.

The result shows that dust particles identification can 
not be solved completely by detecting junction points, so a further determination is needed for images with junction points. After analyzing regions near junction points, a discovery can be made that gray level of dust particles changes significantly, and the gray level value is great in that most dust particles exist on insulating substrate. According to great gray level value and obvious gray level change of dust region, mean value, range, variance and weber coefficient are applied to present characteristics of dust particles. The template to extract features is shown in Figure 10, and the red square presents junction point detected using the method mentioned before. The definition of mean value, range, variance and weber coefficient is list specifically below.

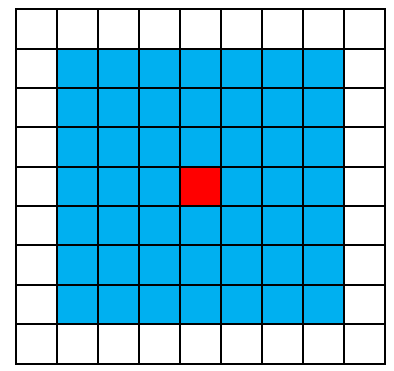

Figure 10. Template.

The mean value of the region for extracting features is defined as

$$
\bar{P}=\frac{1}{n} \sum_{i=1}^{n} P_{i}
$$

$P_{i}$ presents gray level value of pixel point in region, $n$ presents the number of pixel points, and $n=81$ here.

The range of the region for extracting features is defined as

$$
R=P_{\max }-P_{\text {min }}
$$

$P_{\max }$ presents the maximum gray level value of pixel point in region, $P_{\min }$ presents the minimum gray level value of pixel point in region.

The variance of the region for extracting features is defined as

$$
D=\frac{1}{n} \sum_{i=1}^{n}\left(P_{i}-\bar{P}\right)^{2}
$$

$P_{i}$ presents gray level value of pixel point in region, $\bar{P}$ presents the mean value of the region, $n$ presents the number of pixel points, and $n=81$ here.

The weber coefficient ${ }^{[13]}$ of the region for extracting features is defined as

$$
\bar{W}=\frac{1}{n} \sum_{i=1}^{n} W_{i}
$$

$W_{i}=\Delta\left(P_{i}\right) / P_{i}, \Delta\left(P_{i}\right)=\sum_{k=1}^{m}\left|P_{i, k}-P_{i}\right|, W_{i}$ presents the weber coefficient of pixel point, $n$ presents the number of pixel points, and $n=49$ here, which presents the $7 \times 7$ blue region in the template. $P_{i}$ presents gray level value of pixel point in region, $\Delta\left(P_{i}\right)$ presents the absolute value of pixel gray difference with its neighborhood pixels, $P_{i, k}$ is the $k$ neighbor pixel point of $P_{i}, k$ is the number of neighbor pixel points, and $k=8$ here, because of using eight neighborhood.

SVM is a data mining approach on the basis of statistiacal learning theory, which can solve regression problems(time series analysis) and pattern recognition problems (classification and discrimination) ${ }^{[14]}$. In other words, SVM is a process of machine learning, which looks for a classification hyperplane in high dimensional space to separate date points of different categories, and maximize the largest distance between points of different categories $^{[15]}$.

According to the theory of SVM, the dust particles identification is classified as a binary classification problem in this paper, one kind is dust particles image class, and the other kind is non-dust particles image class which includs normal and defect images. The mean value, range, variance and weber coefficient of an image are defined as the average of eigenvalues of regions for extracting features. Given the training sample set $\left(\mathbf{x}_{i}, y_{i}\right), i=1,2, \ldots, I, \quad \mathbf{x} \in R^{n}, y \in\{ \pm 1\}$, hyperplane is $(\mathbf{w} \cdot \mathbf{x})+b=0$. In order to make the classification plane classify samples correctly, and have classification interval, the constraint $y_{i}\left[\left(\mathbf{w} \cdot \mathbf{x}_{i}\right)+b\right] \geq 1, i=1,2, \ldots, I$ requires to be met. The classification interval is $2 /\|\mathbf{w}\|^{2}$. To seek the optimal hyperplane problem is converted to minimize $\|\mathbf{w}\|^{2}$ under constraint.

Dust particles image training sample set is $\left(\mathbf{x}_{m}, 1\right)$, $m=1,2, \ldots, M$, non-dust particles image training sample set is $\left(\mathbf{x}_{n},-1\right), n=1,2, \ldots, N, x$ is an eigenvector including mean value, range, variance and weber coefficient. For forecast sample set $\left(\mathbf{x}_{k}, y_{k}\right)$, $k=1,2, \ldots, K, \quad \mathbf{x} \in R^{n}, y \in\{ \pm 1\}$, the classification is predicted by using the SVM classifier to determine that the sample belongs to dust particles $(y=1)$ or non-dust particles $(y=-1)$.

\section{Results}

Using images from the production line, 1807 dust particles images and14652 non-dust particles images of nine image sets that are needed to be verified are achieved. Automatic feature extraction program is written to collect sample sets of two kinds. According to the optimum principle that positive and negative samples are $1: 1$, positive and negative samples are selected randomly to establish the training sample set, cross validation method is adopted to determine the parameters for the training process ending up with a better classifier model, which is used to predict and classify test images.

Automatic detection program is written with junction point detection and SVM prediction to classify images from image set IPC00009 which is to be verified, test results are shown in Table 1. The junction point 
detection method can detect $97.7 \%$ of dust particles images, and the SVM can identify $80.9 \%$ of dust particles images on the basic of it. According to it, the method including junction point detection and SVM achieve a $94.8 \%$ correct rate of dust particles images identification.

Table 1.Test result.

\begin{tabular}{|c|c|c|c|c|c|c|}
\hline \multicolumn{2}{|l|}{ IPC00009 } & $\begin{array}{l}\text { Number } \\
\text { /Picture }\end{array}$ & $\begin{array}{c}\text { Dust } \\
\text { Number/ } \\
\text { Picture }\end{array}$ & 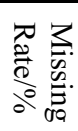 & 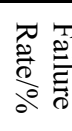 & 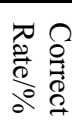 \\
\hline \multicolumn{2}{|c|}{$\begin{array}{l}\text { No-Junction } \\
\text { Point }\end{array}$} & 2710 & 26 & \multirow{3}{*}{$\begin{array}{l}\omega \\
\infty \\
0 \\
0\end{array}$} & \multirow{3}{*}{$\frac{\overrightarrow{0}}{\partial^{\circ}}$} & \multirow{3}{*}{$\begin{array}{l}\stackrel{0}{+0} \\
\stackrel{0}{\circ}\end{array}$} \\
\hline \multirow[t]{2}{*}{$\begin{array}{l}\text { Junction } \\
\text { Point }\end{array}$} & $\begin{array}{l}\text { No- } \\
\text { Dust }\end{array}$ & 1961 & 190 & & & \\
\hline & Dust & 995 & 913 & & & \\
\hline
\end{tabular}

\section{Conclusion}

According to the result above, for dust particles identification of COF, the method proposed in this paper with combination of junction point detection and SVM has certain feasibility, and the method is easy to realize without complex calculation, reference images and image registration. Therefore, it has certain reference significance to solve dust particles interference in COF quality test, and provides a reference for the actual production on the basis of the $94.8 \%$ correct rate.

\section{Acknowledgements}

This research was supported by KGK Precision Electronics Technology Development(Suzhou) Co.Ltd.

\section{References}

1. K. Toyosawa, N. Nakamura, K. Fukuta, Y. Chikawa, COF(Chip-On-Film) Technology for LCD Driver ICs Using Reel-to-Reel System, Sharp Corporation Technical Report 8, 56 (2001)

2. J.Q. Cai, The current status and perspective of COF tape for large scale LCD, Short Comment \& Introduct 6, 10(2012)

3. X.F. Dong, Z.Y. Han, S.Y. Liao, X.X. Yi, Study on Semiconductor Surface Defect Detection Based on Machine Vision, Metrology \& Measurement Technology 5, 22(2014)

4. Y.J. Roh, C.W. Kim, C.O. Jung, D. Jeong, Defect Classification Using Bayesian Approach for Tape Substrate Inspection System, Proceedings of the 17th World Congress on The International Federation of Automatic Control, Seoul, Korea, July 6-11, 8177(2008)

5. R. K, D.K. M, V.K. K, S.P. Nath, Optical Pattern Inspection for Flex PCB - Challenges \& Solution, Proceedings of the 17th World Congress on The International Federation of Automatic Control, Seoul, Korea, July 6-11, 8196(2008)

6. C.T. Liao, W.H. Lee, S.H. Lai, A Flexible PCB Inspection System Based on Statistical Learning, J Sign Process Syst 67, 279(2012)
7. J.S. Kim, COF Defects Detection and Classification System Based on Reference Image, Journal of the Korea Institute of Information and Communication Engineering 17, 1899(2013)

8. P. Jiang, X. B. Xu, M.Fang, Study and Implementation of Skeleton Extraction Algorithm Based on Morphology, Computer Application 23, 136(2003)

9. L. Lam, S. W. Lee, C. Y. Suen, Thinning Methodologies-A Comprehensive Survey, IEEE TRANSACTIONS ON PATTERN ANALYSIS AND MACHINE INTELLIGENCE 14, 869(1992)

10. L. Yu, G.W. Wang, Fast Thinning Algorithm of Binary Images, Science and Technology Vision 5, $100(2012)$

11. W. Yang, K. Guo, Y.K. Wei, An Efficient Index Thinning Algorithm of Fingerprint Image Based on Eight Neighbourhood Points, JOURNAL OF SICHUAN UNIVERSITY OF SCIENCE \& ENGINEERING (NATURAL SICENCE EDITION) 21, 61(2008)

12. A.B. Naeem, H. Allan, Granulometry based Detection of Junction and End Points in Patent Drawings, 7th International Symposium on Image and Signal Processing and Analysis, Dubrovnik, Croatia, September 4-6, 307(2011)

13. X.L. Zou, G.C. Feng, Weber's Law-based Transition Region Extraction and Thresholding, Science Technology and Engineering 13, 4217(2013)

14. S.F. Ding, B.J. Qi, H.Y. Tan, An Overview on Theory and Algorithm of Support Vector Machines, Journal of University of Electronic Science and Technology of China 40, 2(2011)

15. J.B. Xie, Visual Machine Learning 20, Tsinghua University Press, 88(2015) 